\title{
A demonstration of endogenous modulation of unilateral spatial neglect: The impact of apparent time-pressure on spatial bias
}

\author{
MELANIE S. GEORGE, ${ }^{1,2}$ JACKIE S. MERCER,${ }^{3}$ ROBIN WALKER, ${ }^{2}$ AND TOM MANLY ${ }^{1}$ \\ ${ }^{1}$ UK Medical Research Council Cognition and Brain Sciences Unit, Cambridge, Cambridgeshire, United Kingdom \\ ${ }^{2}$ Royal Holloway, University of London, London, United Kingdom \\ ${ }^{3}$ Cambridgeshire Primary Care Trust, Cambridge, Cambridgeshire, United Kingdom
}

(Received March 28, 2006; Final Revision June 18, 2007; Accepted June 18, 2007)

\begin{abstract}
Unilateral neglect, a lack of awareness for one side of space, is a common and debilitating consequence of stroke. Previous work has identified a relationship between enduring left neglect and diminished general alertness and shown that increasing alertness can temporarily reduce the severity of the spatial bias. In that research, alertness was modulated by loud tones or with pharmacological stimulants. Here we examine whether cognitive, endogenously driven changes can produce similar short-term improvements. Time-pressure is associated with increased subjective arousal and increased activation in cortical regions associated with alertness. Here five patients completed a spatial cancellation task with and without instructions regarding a time limit. Significant reductions in neglect severity were observed when patients believed that they were acting under time-pressure, despite the conditions being equivalent in the actual (unlimited) time available. Functional imaging work has highlighted the secondary effects that damage to networks mediating alertness can have on structurally intact spatial systems. The results here suggest that activation of presumably spared function in these damaged networks can induce moment-by-moment changes in spatial function and, crucially, that this can be achieved using entirely endogenous means. (JINS, 2008, 14, 33-41.)
\end{abstract}

Keywords: Rehabilitation, Neuropsychological assessment, Arousal, Visual attention, Stroke, Cancellation tests

\section{INTRODUCTION}

Unilateral spatial neglect is an acquired difficulty in detecting, localizing, or acting on objects on one side of space that cannot be fully explained by basic visual loss (Brain, 1941). Such spatial biases may have profound functional consequences such as missing food on half of the plate, missing words from one side of a page and failure to use left limbs (e.g., Robertson \& Halligan, 1999). Chronic neglect is associated with slowed recovery and appears to have significantly greater impact on functional disability and family burden than would be predicted by simple measures of severity such as lesion extent (Buxbaum et al., 2004; Paolucci et al., 2003).

Correspondence and reprint requests to: Tom Manly, MRC Cognition and Brain Sciences Unit, Box 58 Addenbrooke's Hospital, Cambridge CB2 2QQ United Kingdom. E-mail: tom.manly@mrc-cbu.cam.ac.uk
Neglect is a surprisingly common disorder. Within the first 3 days following a stroke, up to $82 \%$ of patients with right hemisphere $(\mathrm{RH})$ damage are reported to ignore some information from the left side of space. Approximately $65 \%$ of patients with left hemisphere (LH) damage similarly ignore some contralesional information. However, almost all patients with chronic neglect have RH lesions and ignore information in left space (Stone et al., 1993). This imbalance has led to speculation that other capacities disproportionately supported by the RH, if damaged, may have a particular role in maintaining the disorder (Heilman \& Watson, 1987; Husain \& Rorden, 2003; Posner, 1993; Robertson \& Manly, 1999). Among the prime suspects are systems implicated in the maintenance of alertness.

The disproportionate role apparently played by the $\mathrm{RH}$ in maintaining a "ready-to-respond" alert state was first noted in neuropsychological studies in the 1960s (e.g., De Renzi \& Faglioni, 1965) and has subsequently received a 
degree of convergent support from focal lesion and functional imaging studies (Pardo et al., 1991; Sturm et al., 1999; Sturm \& Willmes, 2001; Wilkins et al., 1987). If damage to systems mediating alertness exacerbate or impede the recovery from neglect (rather than simply co-occurring with neglect), it would be expected that patients who show persistent forms of the spatial disorder would be more likely to have difficulties in this respect. Work by Robertson et al. (1997) and Samuelsson et al. (1998), in which deficits in nonspatial sustained attention tasks were disproportionately common among patients with chronic neglect, supported this view. More compelling evidence of a specific link has come from studies that have sought to directly modulate alertness levels. In 1998, Robertson et al. demonstrated that exposure to loud tones immediately before making a spatial judgment significantly ameliorated, abolished or, in some cases, even reversed patients' neglect on that subsequent judgment - a finding that was attributed to exogenously driven increases in alertness following the tone (Robertson et al., 1998). Temporary improvements in neglect following the administration of stimulants (Hurford et al., 1998) and increases in neglect with sedation (Lazar et al., 2002) are also consistent with this view. Intriguingly, analogous patterns of alertness-spatial attention interactions have now been reported in healthy adults (Fimm et al., 2006; Manly et al., 2005b), healthy children (Dobler et al., 2005), and children with developmental disorders, particularly attention deficit hyperactivity disorder (Dobler et al., 2003; Manly et al., 2005a, 1997; Nigg et al., 1997; Sheppard et al., 1999).

A recent functional imaging study with patients suggests a plausible neural basis for the influence of changes in alertness on spatial function (Corbetta et al., 2005). The authors note that brain regions that might be good candidates for explaining neglect type phenomenon-in that they are activated as healthy participants perform lateralized visual attention tasks - are distinct from those regions in which damage most commonly leads to neglect. Specifically, spatial cueing tasks tend to activate frontal and parietal regions that are more dorsal than the right inferior parietal, ventral frontal, and superior temporal regions typically implicated in neglect. Rather than spatial function, these regions are more commonly activated in tasks requiring target detection, alertness, or vigilance. They argued that if this "alertness network" normally interacts with spatial areas, damage may cause relative deactivation in spatial regions, despite their structural integrity. The results from the patient study were consistent with this view. When tested on a cueing task in the immediate post-stroke period, spatial areas in the right hemisphere were relatively deactivated, the degree of deactivation being correlated with the level of impairment on the task. They also noted that in inferior parietal areas, the deactivation of the right hemisphere was accompanied by increased activation in analogous left hemisphere regions. In an elegant aspect of the design, the same patients were again scanned 35 weeks later when significant recovery had occurred. The recovery was associated with reactivation of the structurally intact spatial network and-for the parietal regions-relative deactivation of competitive regions in the LH.

If damage to the more ventral alertness network can have this effect over spatial function-and damage to that system is not absolute-then precisely the type of alertnessdependent modulation of spatial attention seen in the studies highlighted above would be expected. In addition to seeing the changes highlighted by Corbetta et al. over months of recovery, one might also see effects over seconds contingent upon short-scale shifts in alertness. One route for that modulation might be ascending activation from reticular brain stem structures consequent upon, for example, a loud tone (Robertson et al., 1998). Another might be via the effects of stimulant medication (Hurford et al., 1998). An interesting question, that we address here, is whether the alertness network can be modulated in a top-down manner with analogous benefits for spatial function?

There is currently only one suggestion that this may be the case. In 1995, Robertson et al. trained eight patients with neglect in a self-alerting procedure. The idea was for patients to experience an exogenously induced alert state driven by the trainer banging loudly on a desk-and to associate this with a particular self-instruction ("attend!"). By systematically pairing the two and subsequently fading the trainer's role, it was hypothesized that the patients could induce this state themselves with an internal subvocal cue. The training, which lasted 5 days, produced statistically significant improvements in their neglect. One difficulty in fully interpreting the mechanisms of change in this pioneering study comes from its attempt to establish a durable effect. To this end, assessment of the effectiveness of the intervention occurred at least 24 hours after the previous "self-alerting" session. Given the covert nature of the patients' self-alerting, it is not entirely clear whether they were actively alerting themselves during the assessment, whether they had generally raised levels of alertness contingent upon the previous training, or whether some rather less specific process (e.g., relating to the testing environment) was at work. The aims to the current study were to establish whether, in principle, an endogenous alerting procedure was sufficient to provoke short-term changes in spatial function in patients with neglect. To do this, we sought to rely not on the patients alerting themselves as a deliberate act, which may be open to different interpretations and strategies, but rather in inducing a belief that is indirectly associated with increased alertness.

A belief that is known to be probabilistically associated with increased alertness is that one is acting under timepressure. Giving people tasks in which the available time is perceived to be insufficient has been used as a relatively benign means of creating "high arousal" conditions (Steinsmeier-Pelster and Schürmann, 1993) and indeed has been associated with increased activation in frontal (and particularly right frontal) regions (Slobounov et al., 2000) implicated in other studies of alertness. The simple aim of this study was, therefore, to establish whether the belief that they were acting under time-pressure would be suffi- 
cient to significantly improve the spatial bias shown by patients with unilateral neglect.

The experiment consisted of four consecutive administrations of a cancellation test in which participants were asked to find and cross out visual stimuli scattered across a page. The first administration-in line with the standard clinical instructions - had no time limit. With the second administration our aim was to establish a realistic sense of time-pressure by (1) saying that there was a time cut-off (although not what the time-limit was) and (2) stopping the task after just 10 seconds - that is, long before anyone could complete the task. For the third administration, patients were again told that there was a time-limit. In fact, aside from this information, the task was administered in precisely the same way as the first condition. Only 5 seconds after patients had already indicated that the task was complete were they told that the "time-limit" had been reached. In the final administration, we returned to standard conditions, explicitly saying that the patients had as much time as was needed. We hypothesized that patients would show less spatial neglect when acting under the belief that there was time-pressure than under either standard administration - this being important to rule out, for example, simple practice effects.

A secondary hypothesis was that, when acting under perceived time-pressure, the patients would find targets more quickly than under the standard open-ended conditions. It may appear a rather obvious prediction that time-pressure tends to make people perform a given task more quickly, but, nevertheless, this prediction is useful if we are to suggest that at least one operational definition of "alertness", that is, a readiness to respond that increases the speed of subsequent responses (Posner, 1980), was increased by our manipulation.

\section{METHOD}

\section{Participants}

Following approval from the Local Research Ethics Committee, five participants (four women and one man; mean age, 70 years; $S D, 11.37$; range, 58 to 84 ) gave written informed consent for participation in the study. All had suffered a RH stroke at least 3 months previously and been diagnosed with unilateral spatial neglect. These diagnoses were confirmed at the time of assessment on a range of measures. Four of the participants were right-handed and one originally left-handed (although now using her right hand due to hemiplegia).

Demographic, lesion information and background neuropsychological test performance for the participants are given in Table 1. As can be seen in the table, all patients had right hemisphere lesions which were, for the most part, extensive. The group was, in general, of average or above average premorbid intellectual ability as estimated on the National Adult Reading Test (Nelson, 1991). Similarly, although one patient's (S.U.) scores fell at the 10th percentile, the participants were within the normal range on a test of current intellectual function. In terms of spatial function, the patients showed a mean deviation of $36.50 \mathrm{~mm}$ ( $S D$, 23.36) on the Behavioural Inattention Test line bisection task (Wilson et al., 1987), all scores exceeding the 99th percentile on this measure. Although this task gives a somewhat different estimate of neglect severity from performance on the Star Cancellation task, averaging across the measures would place the patients in order S.U., K.Q., T.I., D.C., and T.T. in terms of overall bias (most to least). Three patients (T.I., D.C., and S.U.) showed evidence of visual

Table 1. Demographic, lesion, and background neuropsychological information on the participants

\begin{tabular}{|c|c|c|c|c|c|}
\hline \multirow[b]{2}{*}{ Characteristics } & \multicolumn{5}{|c|}{ Patient participants } \\
\hline & 1 T.I. & 2 D.C. & 3 T.T. & 4 K.Q. & 5 S.U. \\
\hline Age (yr) & 58 & 81 & 67 & 84 & 63 \\
\hline Sex & $\mathrm{F}$ & $\mathrm{F}$ & $\mathrm{F}$ & $\mathrm{F}$ & M \\
\hline Time since stroke (months) & 6 & 9 & 4 & 4 & 3 \\
\hline $\begin{array}{l}\text { Characterization of lesions from } \\
\text { CT interpretation }\end{array}$ & $\begin{array}{l}\text { Extensive right } \\
\text { middle cerebral } \\
\text { artery (MCA) } \\
\text { infarct with no } \\
\text { hemorrhage }\end{array}$ & $\begin{array}{l}\text { Right basal } \\
\text { ganglia infarct, } \\
\text { evidence of } \\
\text { paraventricular } \\
\text { white matter } \\
\text { ischemia }\end{array}$ & $\begin{array}{l}\text { Large right } \\
\text { hemisphere } \\
\text { hemorrhage } \\
\text { resulting in } \\
\text { right posterior } \\
\text { temporal and } \\
\text { parietal damage } \\
\text { (including to the } \\
\text { inferior parietal } \\
\text { lobe) }\end{array}$ & $\begin{array}{l}\text { Large right } \\
\text { hemisphere infarct, } \\
\text { including lesion } \\
\text { extending to } \\
\text { inferior parietal } \\
\text { and posterior } \\
\text { temporal lobe }\end{array}$ & $\begin{array}{l}\text { Right frontal } \\
\text { lacunar type } \\
\text { infarct; lesion } \\
\text { deep-seated in } \\
\text { white matter } \\
\text { of the frontal } \\
\text { lobe }\end{array}$ \\
\hline Years of education & 12 & 9 & 11 & 16 & 10 \\
\hline Estimated Premorbid IQ (from NART) & 113 & 100 & 105 & 117 & 89 \\
\hline $\begin{array}{l}\text { Current Intellectual functioning from } \\
\text { VESPAR-percentiles }\end{array}$ & $74^{\text {th }}$ & $44^{\text {th }}$ & $54^{\text {th }}$ & $82^{\text {nd }}$ & $10^{\text {th }}$ \\
\hline $\begin{array}{l}\text { Line bisection (mean deviation } \\
\text { from center in } \mathrm{mm} \text { ) }\end{array}$ & 33.00 & 17.80 & 13.70 & 47.00 & 71.00 \\
\hline
\end{tabular}


extinction in standard confrontation testing. K.Q. and T.T. were unable to detect the left-sided stimuli in this task, even when presented in isolation-consistent with hemianopia.

\section{Measures and Apparatus}

The standard version of the Star Cancellation test shows 56 small stars distributed across a landscape orientation white A4 paper sheet among a larger star, words, and letter distractors. To reduce practice effects attributable to remembering the specific locations of targets, 10 variants of the original sheet were generated by first scanning the sheet and then repositioning targets and distractors using photo editing software. While the precise locations of the targets and distractors varied between the sheets, the use of an eight-box grid during editing ensured that distribution across the sheet was even (i.e., left-right and upper-lower).

The Star Cancellation sheets were presented on a bespoke milled aluminum tray $(420 \mathrm{~mm} \times 30 \mathrm{~mm})$ with a central depression $(29.6 \mathrm{~mm} \times 21 \mathrm{~mm})$ coated with a rubber sheet. The depression in the tray was such that a standard A4 paper sheet would sit flush with the surface while being held securely in position. In addition, the apparatus also served as the base for a video stand. Specifically, a pole was attached to base points situated at the left and right sides of the apparatus. A video camera on a universal joint mount was thus afforded a clear view of a held pen marking the sheet-whether a participant was left- or right-handed.

\section{Procedure}

\section{General procedure}

Participants were tested in their own homes. The cancellation sheets were presented using the apparatus described above placed centrally on a table at the patient's midline. No restrictions were placed on their eye or head movements during testing, although participants were discouraged from moving the sheet, and from leaning excessively to one or other side. Performance of all cancellation sheets was video recorded for subsequent scoring.

\section{Baseline I: Standard Star Cancellation administration}

Participants were first administered the Star Cancellation test in essentially the standard way (Wilson et al., 1987). Specifically 1 of the 10 variants of the star cancellation sheet was selected at random and placed on the tray in front of the participants. The participants were told that the page contained stars of different sizes. They were asked to look the page carefully to cross out all of the small stars. Crucially, in terms of the time-pressure manipulation, in this standard administration participants are asked to go "through the page and cross out the small stars without missing any of them and tell me when you have finished."

\section{Establishing Time-Pressure- 10-Second Condition}

Following the first standard condition, a second sheet was selected at random from the 10 alternative versions and placed on the aluminum tray. Participants were told "Last time I was interested in how many of the small stars you could find; this time I want to see how many you can find within a time limit. I will not tell you how long the time limit is but I will just say 'stop', so please find and mark the small stars as quickly as you can after I say 'go'. I will begin counting down when you have 5 seconds left. You will not know when that will be, so please keep going until I say 'stop'". Five seconds after the participants had started the task, the researcher counted aloud " $5,4,3,2,1$ " and then said, "Stop"- - the total duration of the trial being $10 \mathrm{sec}-$ onds. This condition was purely to lend credibility to the idea of a time-limit and did not contribute to subsequent analyses.

\section{Apparent Time-Pressure Condition}

The instructions for the next trial were identical to the previous condition. Participants were again asked to cross out as many of the stars as possible within an unspecified time limit and were told that the last 5 seconds would be counted down for them. However, no fixed time limit was imposed. When a participant stated that $\mathrm{s} / \mathrm{he}$ had completed the task, the researcher began counting down the last 5 seconds and then said "stop."

\section{Baseline II: Standard Administration}

The final trial reverted to the standard administration, as described above. The participants were told "this time I just want you to focus on finding as many small stars as possible. There is no time limit so go through this page and cross out all the small stars without missing any of them, and tell me when you think that you have found them all."

\section{Procedure for Deriving Timing Scores From the Video Recordings}

From the video recordings, the time taken to find each target in each cancellation performance was subsequently scored by two independent raters. One rater was blind to which patient's performance was being viewed (only the hand and arm are visible) and from which condition the recording came. Time zero was set at the first contact of the pen at the outset of the first cancellation mark. The time of each subsequent cancellation was again defined by the first contact of the pen at the outset of each mark. Variations in lighting conditions (e.g., shadows obscuring the pen in some parts of the sheet), occasional camera shakes, and so on mean that some interpretation is periodically required in deciding when the pen has first made contact. It 
is also possible, of course, that raters can make transcribing and other errors in their work. Accordingly, we consider the reliability of the scores further in the results below.

\section{RESULTS}

\section{Neglect Severity}

An estimate of neglect severity at the time of assessment can be derived from initial performance on the Star Cancellation test (i.e., the first standard clinical administration). As a group, the patients detected a mean of 43.80/56 targets $(S D, 5.45)$. The mean detection rates for the left and the right sides of the sheet were 18.40/28 ( $S D, 3.21)$ and $25.40 / 28(S D, 3.21)$, respectively. All patients would, therefore, have fallen below the clinical cut-off criteria (Wilson et al., 1987). Whereas, as is common, performance was far from perfect on the right of the sheet, all participants showed the expected bias favoring right-sided detection [repeated measures analysis of variance (ANOVA) comparing detection on the left and the right; $F(1,4)=21.304, p=.01]$. The relative performance of the patients on this and the subsequent sheets are shown in Figure 1.

\section{Modulation of Neglect Severity by Apparent Time Pressure}

To examine the effect of apparent time-pressure a repeated measures ANOVA was performed on the dependent vari- able of targets detected. The factors were side (number of targets found on the left and the right side of the sheet) and condition (averaged performance for baseline conditions 1 and $2 v s$. performance under apparent time-pressure). In contrast to the examination of the first baseline condition presented above, over the three administrations there was only a modest trend toward a difference in target detection between the left and the right of the sheet $[F(1,4)=5.29$, $p=.083$; average target detection on the left $=21.13, S D$ 5.11 ; average target detection on the right $=26.33, \mathrm{SD}$ 2.024]. There was also a modest trend toward an overall effect of condition $(F(2,8)=5.61 ; p=.07)$, with more targets being detected overall under apparent time-pressure (25.30; SD , 3.68) than under the baseline conditions (22.95; $S D, 4.56)$. Crucially, however, there was a statistically significant interaction between side and condition $(F(2,8)=$ 20.17; $p=.01)$. Figure 1 shows the target detection rates for each participant (and the averaged group results) under baseline I, time-pressure condition and baseline II for the left and right sides of the sheet, respectively. As is illustrated, every participant showed an increase in target detection on the left in the time-pressure condition relative to baseline I (the improvements ranging between 7 and $75 \%$ increases on the initial score). All but one of the participants then showed a decline from the time-pressure to the second baseline condition (reductions of between 8 and $31 \%$ of the time-pressure total). This finding suggests that the time-pressure effect does not simply reflect practice. Look-

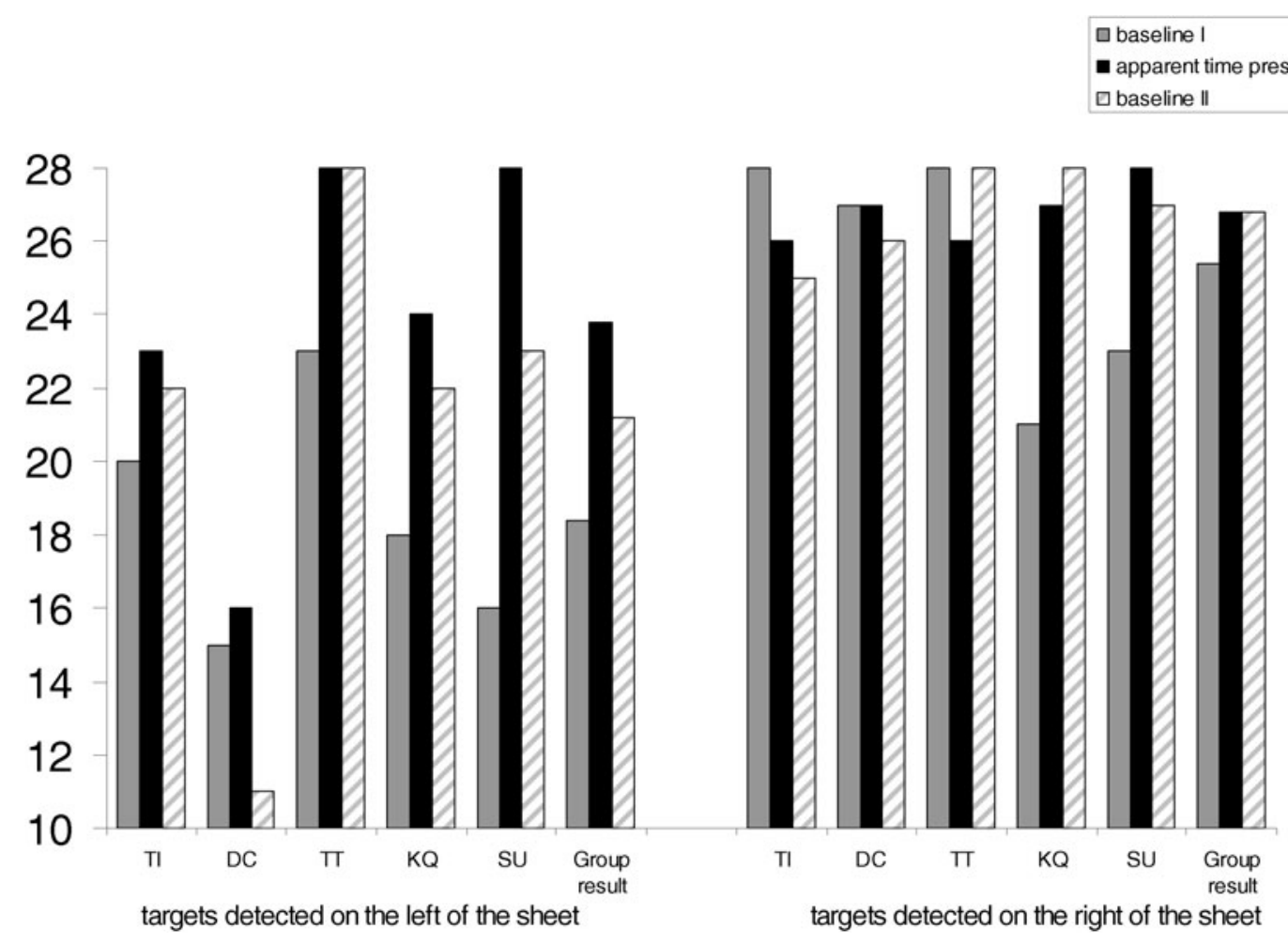

Fig. 1. Targets detected on the left and the right sides of the sheet during baseline I, apparent time-pressure, and baseline II shown for each participant and the group. 
ing at performance on the right side of the sheet, no such consistent pattern is apparent.

\section{The Speed With Which Participants Cancelled Targets in Each Condition}

Equating speed across the conditions is not entirely simple. One measure would be the total time taken to self-reported completion of the task. This is however complicated by the accuracy of performance - a patient with very severe neglect may find very few targets on the extreme right-side of the page and, as a consequence, "complete" the task very quickly. If time-pressure was successful, as hypothesized, in reducing spatial neglect it could well lead to a longer time to self-reported completion. An alternative, that we adopt here, was to find the minimum number of targets found by any patient in any condition and to take the time required to find that number of targets by the patients in each of the conditions. It turned out that the fewest targets detected by any of the patients was 37. The degree of agreement between the two independent raters over the 15 video recordings at approximately the time at which the 37 th cancellation occurred was very high, $(n=15$, Pearson's $r=0.99, p<$ $.001)$ with the averaged discrepancy across the 15 videos being only $800 \mathrm{~ms}$.

The time required to find the first 37 targets by each patient was entered into a repeated-measures ANOVA with the factor of condition (baseline I, apparent time-pressure and baseline II). The results showed a statistically significant effect of condition with the time taken in baseline I being $73.40 \mathrm{~s}(S D, 21.23)$, reducing by an average of $40 \%$ to $44.00 \mathrm{~s}$ in the apparent time-pressure condition ( $S D, 17.24)$ and then increasing again to $55.27 \mathrm{~s}(S D, 13.48)$ in the second baseline condition $[F(2,8)=9.05, p=.009]$. Post hoc analysis using Least Significant Difference implemented in SPSS version 11 revealed a significant reduction from the first baseline to the time-pressure condition ( $p=$ $.02)$ and a significant reduction in the second baseline condition relative to the first ( $p=.03$ ). The difference between the time-pressure condition and the second baseline was not, however, statistically significant at the 37th target ( $p=$ .16). Figure 2 shows the averaged time-per-target at each cancellation point for the patient group, illustrating a very consistent pattern from the outset of the task with the first baseline being the slowest, the apparent time-pressure being the fastest, and the second baseline sitting somewhere in-between. That there is some slowing in the second baseline condition suggests that the "speeding" in the timepressure condition is not simply a practice effect. Whether the relative speeding in the second baseline compared with the first reflects a practice effect or a "carry-over" from the time-pressure conditions is less clear.

\section{DISCUSSION}

In this study, five patients with spatial neglect were asked to perform the Star Cancellation test under two conditions. In the baseline condition, the standardized clinical administration was used. In the experimental condition, the patients were told that there was a time limit. With the exception of a brief condition designed to lend credibility to the notion of a time-limit, the administration of the conditions was in

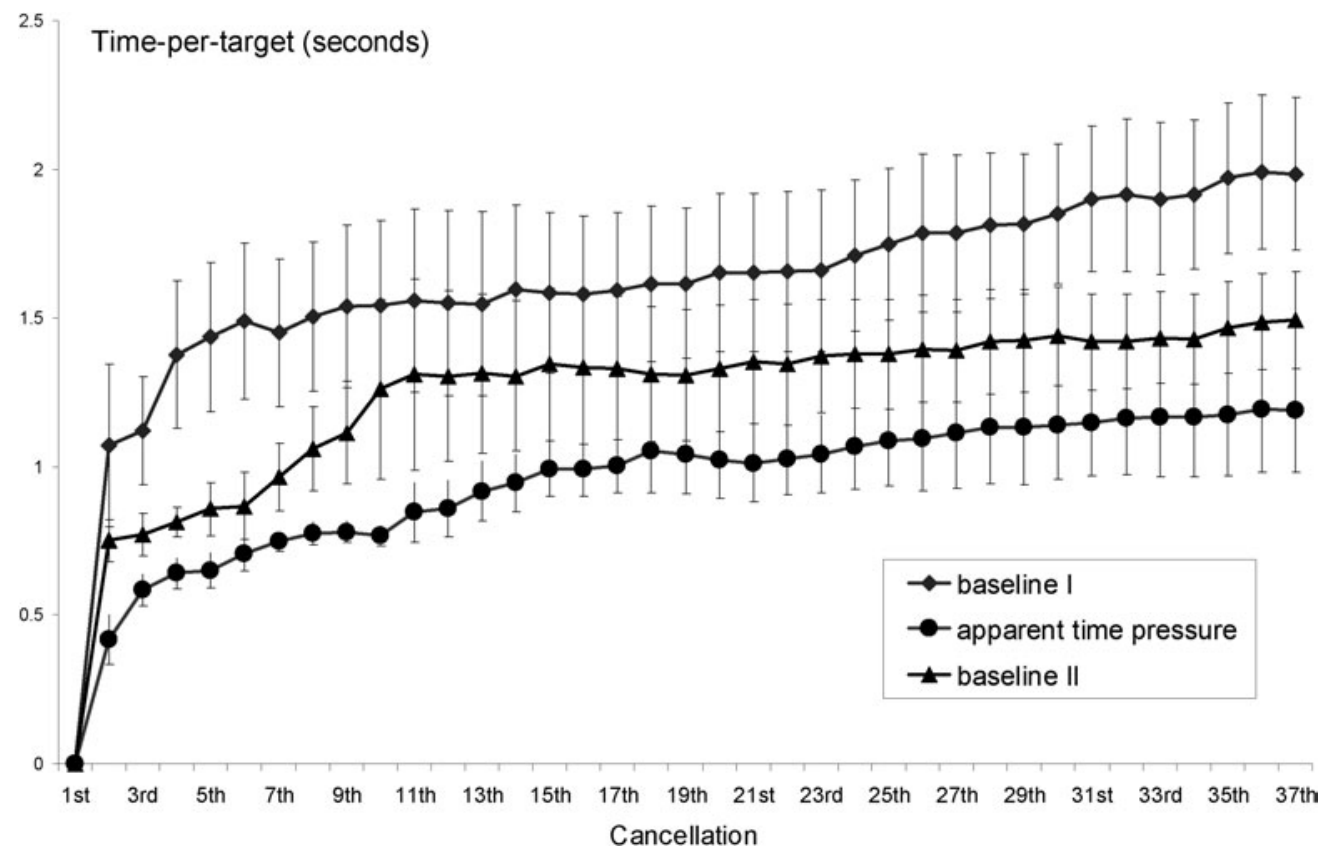

Fig. 2. Speed of performance under the two baseline and apparent time-pressure conditions. The averaged time-pertarget in seconds (time from the onset of the task at which each subsequent target was canceled divided by the total number of targets canceled at that point) averaged across the five patient participants (error bars, standard error) 
fact identical in terms of the goals of the task and the unlimited time available. When the patients believed that they were under time-pressure, however, they showed significantly less neglect of left space. Immediately afterward, in a second administration when we again made it explicit that there was no time-limit, this improvement was no longer evident. Given this balance in the design, it is reasonable to conclude that perceived time-pressure is associated with reduced spatial neglect, at least in the patients tested here.

The results are consistent with the hypothesis that a sense of time-pressure would induce a heightened state of arousal/ alertness, which has previously been associated with a reduction in spatial neglect (Dobler et al., 2003; Hurford et al., 1998; Robertson et al., 1998). The increased speed of target detection as well as reduced bias in the apparent timepressure condition is consistent with that view. This speeding does not appear to be a trivial consequence of the reduction in neglect (for example, targets on the left being easier to "see" and, therefore, cancelled more quickly); it is apparent from the outset of the task when patients are typically canceling targets at the extreme right side of the sheet (in this group, 96\% of the first 20 targets cancelled were on the right). Because the external conditions were identical across the three administrations (i.e., no sudden changes in lighting, no loud noises), it is possible to interpret the effect as stemming from a cognitively driven change in alertness induced by the participants' beliefs.

As discussed, the finding is consistent with the right hemisphere having a particular role in developing and maintaining an alert state and the view that such increases assist it in resisting competitive inhibition from the left hemisphere. The recent functional imaging study by Corbetta and colleagues offers a plausible neural basis for such effects (Corbetta et al., 2005). As outlined in the introduction, they suggested that damage to a more ventral frontal and temporoparietal network (associated with, among other things, speeded target detection) had knock-on deactivating effects on more dorsal spatial networks that may be structurally intact. In turn, at least in parietal regions, this effect appeared to be exacerbated by competitive inhibition from analogous regions in the left hemisphere. In that study, the extent to which this occurred was related to the spatial bias to the right shown by patients. Recovery in the behavioral manifestations of neglect also coincided with the reactivation of this spatial system - presumably attributable to some recovery or reorganization in the damaged "alertness" regions. The current study suggests that this modulation may occur not simply over weeks of the recovery but from momentto-moment, depending on the relative level of activation in spared regions of the damaged system. The patterns of activation that have specifically examined time-pressure would be broadly consistent with this explanation (Slobounov et al., 2000), although clearly direct observation of brain changes in patients under the conditions that we have outlined would be the crucial test of the anatomical aspects of this hypothesis.

Previous studies that have demonstrated experimental modulation of spatial neglect by induced changes in alert- ness have done so with loud tones or stimulant medication. In those cases, it has been argued that the effects may be mediated by increased activity in brainstem reticular structures that disproportionately activate the right hemisphere (Husain and Rorden, 2003; Robertson et al., 1998). This study crucially differs in that the changes in alertness were induced at a purely cognitive level, by the implication of the instructions. Of course, such an effect may well work by means of similar mechanisms to those outlined in other studies, that is, by means of increased activation of the reticular formation and thalamic nuclei. The important aspect is in suggesting that a cognitively mediated top-down signal can produce beneficial changes.

It is important to stress at this stage that, while (apparent) time-pressure benefited performance on a cancellation test without any obvious cost, this finding may not be the case for all tasks. The Star Cancellation measure is relatively simple and has no substantial requirement for sensitive discrimination. In many other activities there is a well established speed-accuracy trade-off. The point here is not so much that time-pressure per se improved performance but that some cognitively mediated change significantly increased patients' awareness of left space. This, in common with the results from previous studies outlined above, has clinical implications. First, the demonstration of a "topdown" cognitive route to reducing neglect through increases in alertness supports the arguments outlined by Robertson and colleagues (1995) and suggests that training in selfmaintaining an alert state has at least the potential to produce benefits. While it may be argued that patients are no more likely to remember to "alert themselves" than they are to remind themselves to look to the left over the long-term (Parton et al., 2004), it is possible that early rehabilitation may be sufficient to boot-strap levels of alertness that will then tend to be maintained by, for example, increased engagement with other activities (see Sturm et al., 2004). A second approach lies in the use of stimulant medication. While there are some preliminary data suggesting improvements in spatial bias (Hurford et al., 1998; Sheppard et al., 1999) further investigations, including with the anti-narcolepsy agent Modafinil appear warranted.

Some caution is needed regarding the generality of the current findings. The patients seen here showed relatively mild neglect on the cancellation test. Whether patients with more severe neglect - and by inference more severe lesions or who are earlier in their recovery-would show similar effects remains to be tested. It is perhaps of note that the patient with the greatest severity, S.U., showed the greatest change with the time-pressure intervention, but this may also reflect something of a ceiling effect on others' performance in this condition.

Aside from specific alertness training and stimulant medication, few would argue that an appropriately stimulating environment in which to recover from stroke is not a good thing. However demonstrations, such as the current study, showing direct benefits of changes in alertness on apparently fixed cognitive deficits should add to this case. Clearly, 
there needs to be some balance between a potentially overstimulating/overpressured setting and patients' desire and need to rest quietly. However, in many services-at least in the United Kingdom - this balance may veer far too much toward the latter. A recent study found that within UK specialist stroke rehabilitation settings, patients spent on average only an hour a day engaged in any form of therapy and $65.33 \%$ of their time interacting with no other person (De Wit et al., 2005). At least in terms of spatial neglect, it seems likely that the alerting qualities of many of the components of dedicated therapy sessions (feedback, collaboratively setting goals, and so on) indeed, any activity at all, may have direct as well as more general benefits.

Although we are certainly not advocating giving patients the impression that they are under time-pressure when they are not, the results here suggest one relatively simple way of assessing the likely effects of increased alertness on a given patient's spatial performance. Asking patients to repeat cancellation or other spatial measures as if under timepressure or as if in competition with others may form a useful behavioral experiment. Positive results could inform clinical strategy (be it pharmacological or behavioral) and be illustrative to patients, staff, and caregivers about conditions that optimize function.

\section{ACKNOWLEDGMENTS}

This research was supported by the UK Medical Research Council. We are grateful to the staff at Addenbrooke's Hospital and the Cambridge Community Stroke Team for their assistance and to the participants and their families for their help. We thank Suzy Blows for her careful proofing and setting of this manuscript.

\section{REFERENCES}

Brain, R. (1941). Visual disorientation with special reference to lesions of the right hemisphere. Brain, 64, 244-272.

Buxbaum, L.J., Ferraro, M.K., Veramonti, T., Farne, A., Whyte, J., Ladavas, E., Frassinetti, F., \& Coslett, H.B. (2004). Hemispatial neglect-Subtypes, neuroanatomy, and disability. Neurology, 62, 749-756.

Corbetta, M., Kincade, M.J., Lewis, C., Snyder, A.Z., \& Sapir, A. (2005). Neural basis and recovery of spatial attention deficits in spatial neglect. Nature Neuroscience, 8, 1603-1610.

De Renzi, E. \& Faglioni, P. (1965). The comparative efficiency of intelligence and vigilance tests in detecting hemispheric cerebral damage. Cortex, 1, 410-433.

De Wit, L., Putman, K., Dejaeger, E., Baert, I., Berman, P., Bogaerts, K., Brinkmann, N., Connell, L., Feys, H., Jenni, W., Kaske, C., Lesaffre, E., Leys, M., Lincoln, N., Louckx, F., Schuback, B., Schupp, W., Smith, B., \& De Weerdt, W. (2005). Use of time by stroke patients: A comparison of four European rehabilitation centers. Stroke, 36, 1977-1983.

Dobler, V.B., Anker, S., Gilmore, J., Robertson, I.H., Atkinson, J., \& Manly, T. (2005). Asymmetric deterioration of spatial awareness with diminishing levels of alertness in normal children and children with ADHD. Journal of Child Psychology and Psychiatry, 46, 1230-1248.

Dobler, V.B., Manly, T., Verity, C., Woolrych, J., \& Robertson, I.H. (2003). Modulation of spatial attention in a child with developmental unilateral neglect. Developmental Medicine and Child Neurology, 45, 282-288.

Fimm, B., Willmes, K., \& Spijkers, W. (2006). The effect of low arousal on visuo-spatial attention. Neuropsychologia, 44, 1261-1268.

Heilman, K. \& Watson, R. (1987). Attention: Behavior and neural mechanisms (Vol. V). Baltimore, MD: American Physiological Society.

Hurford, P., Stringer, A., \& Jann, B. (1998). Neuropharmacologic treatment of hemineglect: A case report comparing bromocriptine and methylphenidate. Archives of Physical Medicine and Rehabilitation, 79, 346-349.

Husain, M. \& Rorden, C. (2003). Non-spatially lateralized mechanisms in hemispatial neglect. Nature Reviews Neuroscience, $18,26-36$.

Lazar, R., Fitzsimmons, B., Marshall, R., Berman, M., Bustillo, M., Young, W., Mohr, J., Shah, J., \& Robinson, J. (2002). Reemergence of stroke deficits with midazolam challenge. Stroke, 33, 283-285.

Manly, T., Cornish, K., Grant, C., Dobler, V., \& Hollis, C. (2005a). Examining the relationship between rightward visuo-spatial bias and poor attention within the normal child population using a brief screening task. Journal of Child Psychology and Psychiatry, 46, 1337-1344.

Manly, T., Dobler, V.B., Dodds, C.M., \& George, M.A. (2005b). Rightward shift in spatial awareness with declining alertness. Neuropsychologia, 43, 1721-1728.

Manly, T., Robertson, I., \& Verity, C. (1997). Developmental unilateral visual neglect: A single case study. Neurocase, 3, 19-29.

Nelson, H.E. (1991). National Adult Reading Test (NART): Test Manual (Revised). Windsor: NFER-Nelson.

Nigg, J.T., Swanson, J.M., \& Hinshaw, S.P. (1997). Covert spatial attention in boys with attention deficit hyperactivity disorder: Lateral effects, methylphenidate response and results for parents. Neuropsychologia, 35, 165-176.

Paolucci, S., Antonucci, G., Grasso, M.G., Bragoni, M., Coiro, P., De Angelis, D., Fusco, F.R., Morelli, D., Venturiero, V., Troisi, E., \& Pratesi, L. (2003). Functional outcome of ischemic and hemorrhagic stroke patients after inpatient rehabilitation a matched comparison. Stroke, 34, 2861-2865.

Pardo, J.V., Fox, P.T., \& Raichle, M.E. (1991). Localization of a human system for sustained attention by positron emission tomography. Nature, 349, 61-64.

Parton, A., Malhotra, P., \& Husain, M. (2004). Hemispatial neglect. Journal of Neurology Neurosurgery and Psychiatry, 75, 13-21.

Posner, M.I. (1980). Orientating of attention. Quarterly Journal of Experimental Psychology, 32, 3-25.

Posner, M.I. (1993). Interaction of arousal and selection in the posterior attention network. In A. Baddeley \& L. Weiskrantz (Eds.), Attention: Selection, Awareness and Control. Oxford: Clarendon Press.

Robertson, I.H. \& Halligan, P.W. (1999). Spatial Neglect: A Clinical Handbook for Diagnosis and Treatment. Hove, UK: Psychology Press.

Robertson, I.H. \& Manly, T. (1999). Sustained attention deficits in time and space. In G.W. Humphreys, J. Duncan, \& A.M. Treisman (Ed.), Attention, Space, and Action: Studies in Cognitive Neuroscience. Oxford: Oxford University Press.

Robertson, I., Manly, T., Beschin, N., Daini, R., Haeske-Derwick, H., Homberg, V., Jehkonen, M., Pezzamiglio, G., Shiel, A., \& Weber, E. (1997). Auditory sustained attention is a marker of unilateral spatial neglect. Neuropsychologia, 13, 1527-1532. 
Robertson, I., Mattingley, J., Rorden, C., \& Rorden, J. (1998). Phasic alerting of neglect patients overcomes their spatial deficit in visual awareness. Nature, 395, 169-172.

Robertson, I.H., Tegner, R., Tham, K., Lo, A., \& Nimmo-Smith, I. (1995). Sustained attention training for unilateral neglect: Theoretical and rehabilitation implications. Journal of Clinical and Experimental Neuropsychology, 17, 416-430.

Samuelsson, H., Hjelmquist, E., Jensen, C., Ekholm, S., \& Blomstrand, C. (1998). Nonlateralized attentional deficits: An important component behind persisting visuospatial neglect? Journal of Clinical and Experimental Neuropsychology, 20, 73-88.

Sheppard, D.M., Bradshaw, J.L., Mattingley, J.B., \& Lee, P. (1999). Effects of stimulant medication on the lateralisation of line bisection judgements of children with attention deficit hyperactivity disorder. Journal of Neurology, Neurosurgery and Psychiatry, 66, 57-63.

Slobounov, S.M., Fukada, K., Simon, R., Rearick, M., \& Ray, W. (2000). Neurophysiological and behavioural indices of time pressure effects on visuomotor task performance. Cognitive Brain Research, 9, 287-298.

Steinsmeier-Pelster, J. \& Schürmann, M. (1993). Information processing in decision making under time pressure: The influence of action versus state orientation. Time Pressure and Stress in Human Judgment and Decision Making, 241-253.

Stone, S.P., Halligan, P.W., \& Greenwood, R.J. (1993). The incidence of neglect phenomena and related disorders in patients with an acute right or left-hemisphere stroke. Age and Ageing, $22,46-52$.

Sturm, W., de Simone, A., Krause, B.J., Specht, K., Hesselmann, V., Radermacher, I., Herzog, H., Tellmann, L., Muller-Gartner, H.W., \& Willmes, K. (1999). Functional anatomy of intrinsic alertness: Evidence for a fronto-parietal-thalamic-brainstem network in the right hemisphere. Neuropsychologia, 37, 797-805.

Sturm, W., Longoni, F., Weis, S., Specht, K., Herzog, H., Vohn, R., Thimm, M., \& Willmes, K. (2004). Functional reorganisation in patients with right hemisphere stroke after training of alertness: A longitudinal PET and fMRI study in eight cases. Neuropsychologia, 42, 434-450.

Sturm, W. \& Willmes, K. (2001). On the functional neuroanatomy of intrinsic and phasic alertness. Neuroimage, 14, S76-S84.

Wilkins, A.J., Shallice, T., \& McCarthy, R. (1987). Frontal lesions and sustained attention. Neuropsychologia, 25, 359-365.

Wilson, B., Cockburn, J., \& Halligan, P. (1987). The Behavioural Inattention Test. Titchfield, Hampshire, UK: Thames Valley. 